\title{
Interstellar Grains in Reflection Nebulae
}

\author{
VLADIMIR VANYSEK \\ Charles University \\ Prague, Czechoslovakia
}

Reflection nebulae are suitable objects for studying the physical $\mathbf{R}_{\text {nature of interstellar grains. Unfortunately, there exist only a few }}$ sufficiently bright objects, the most widely studied being nebula NGC 7023. This nebula has been studied photographically and photoelectrically by many authors, and therefore it is best suited for the verification of some theoretical conclusions. Earlier measurements (refs. 1 to 3) have already shown unambiguously that almost all nebulae exhibit a negative color excess in comparison with the color of the illuminating star. This fact has been verified photoelectrically in reference 4 . In the $B-V$ system the average difference between the color of the star and that of a nebula is $-0.2^{\mathrm{m}}$. These facts have been known for a long time, but the distribution of the color excess in the nebula itself has not been studied very intensively.

In the years from 1961 to 1965 we made a series of photoelectric measurements of the NGC 7023 nebula. Photometric measurements were compared with theoretical models computed for different particles of a dielectric nature. Some results have already been published in reference 5. Recently, these published measurements have been completed by measurements in the ultraviolet spectral region. It appears that considerable differences in the color excess in different regions of the nebula are observable. Nevertheless, there exists an evident decreasing trend of the color excess toward the edge of the nebula. This trend is true particularly for the color excess $U-B$ which, as the $B-V$ color excess, is defined according to the relation

$$
E=(B-V)_{n e b}-(B-V)_{s t}
$$

where $(B-V)_{s t}$ is the color index of the star and $(B-V)_{n e b}$ is the color index of the nebula in the $U B V$ system.

The decrease of the negative color excess toward the edge of nebulae 
also follows from photographic results of reference 6 for NGC 7023 and from those of reference 7 , as well as from the photoelectric measurements in reference 8 of the Hubble variable nebula 2261 in Monoceros.

The increasing trend of blue color of nebulae bearing from the illuminating star to a certain distance, as observed by Hall and Martel, is obviously caused by instrumental scattering of the starlight. However, the "return" of color to the color of the star in the area near the edge of the nebula is caused by the intrinsic nature of the nebular particles.

Measurements can be interpreted by comparison with theoretical models based on the following assumptions:

1. The nebula is rather thin, optically, at greater distances from the star.

2. The gradient of the decrease of the density at greater distances is very slow, and for computational purposes, the average density is assumed to remain constant.

3. The nebula itself can have various forms; for the computation of models, the form of a spherical layer with different thicknesses is assumed. Such a model is very suitable from the mathematical point of view, as relations for analogous models can be deduced from it.

Let a mass of homogeneous density be distributed around an illuminating star in the form of a spherical layer. Consider an element in the direction of observation and of unit cross section and length $\mathrm{d} y$, contain-

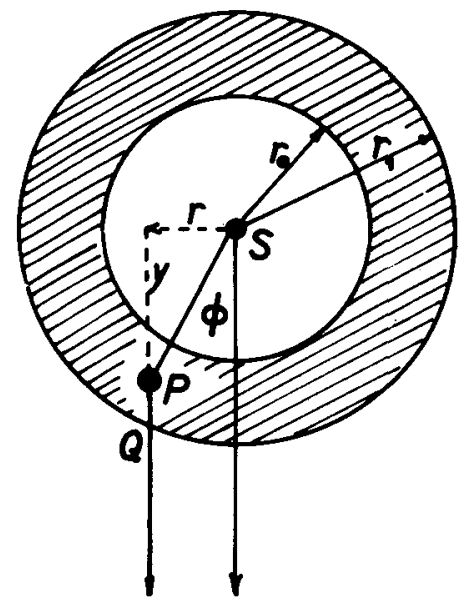

Figure 1.-Model of star with surrounding spherical shell of dust.

ing the point $P$. (See fig. 1.) The scattered light in this element, contributing to the scattered light in the direction $\overline{P Q}$, is given by the expression

$$
d I(r)=I_{s} \omega \mathrm{d} y \mathrm{e}^{-\kappa\left(\overline{S P}-r_{0}+\overline{P Q}\right)} F(\phi)
$$


where

$I_{s} \quad$ energy emitted by the star into a unit solid angle

$\omega \quad$ solid angle under which element is seen from point $S$

$\kappa \quad$ coefficient of absorption

$F(\phi)$ function following from Mie coefficient

Substituting the relations

$$
\begin{aligned}
\overline{S P} & =r \csc \phi \\
\overline{P Q} & =r_{1}^{2}-r^{2}-r_{0} \cot \phi \\
\omega & =\frac{1}{r^{2} \csc ^{2} \phi} \\
y & =r \cot \phi \\
\mathrm{d} y & =r \csc ^{2} \phi \mathrm{d} \phi
\end{aligned}
$$

into equation (2) gives

$$
\mathrm{d} I(r)=I_{s} r^{-1} \exp \left(-\kappa r_{1}^{2}-r-r_{0}\right) \exp (-\kappa r(\csc \phi-\cot \phi) F(\phi) \mathrm{d} \phi
$$

The total brightness of the nebula at a distance $r$ from the center of the nebula $S$ is given by integration

$$
\begin{aligned}
& I(r)=I_{s} \frac{1}{r} \mathrm{e}^{-\kappa\left(\sqrt{r_{1}^{2}-r^{2}}-r_{0}\right)}\left[\int_{\beta \sin ^{-1} r / r_{1}}^{\sin ^{-1} r / r_{0}} \mathrm{e}^{-\kappa r(\csc \phi-\cot \phi)} F(\phi) \mathrm{d} \phi ;\right. \\
& \left.\quad+\int_{\pi-\sin ^{-1} r / r_{0}}^{\pi-\sin ^{-1} r / r_{1}} \mathrm{e}^{-\kappa r(\csc \phi-\cot \phi)} F(\phi) \mathrm{d} \phi\right] \quad \text { for } r<r_{0} \\
& I(r)=I_{s} \frac{1}{r} \mathrm{e}^{-\kappa r\left(\sqrt{r_{1}^{2}-r^{2}}-r_{4}\right)} \int_{-\sin ^{-1} r / r_{1}}^{\pi-\sin ^{-1} r / r_{1}} \mathrm{e}^{-\kappa r(\csc \phi-\cot \phi)} F(\phi) \mathrm{d} \phi
\end{aligned}
$$

for $r \geqslant r_{0}$.

It is evident that the second of equations (5) is actually the formula for the spherical nebula. Analogous relations hold for other models.

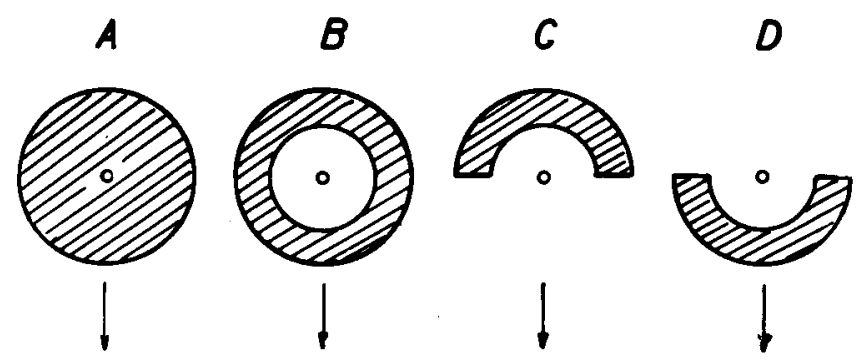

Figure 2.-Models of reflection nebulae. 
On the basis of these relations, the following models (see fig. 2) of nebula have been used for computation of the theoretical color excess $E(r)$ which depends on the distance $r$ :

A a spherical nebula

B a basic model; i.e., the nebula in the form of a spherical layer

C a nebula in the form of a curved layer with the illuminating star before the nebula

D a model analogous to model $\mathrm{C}$ with the illuminating star behind the nebula.

The effective radius of an actual nebula (NGC 7023) $r_{1}$ is assumed to be 720 seconds of arc. The size-distribution function

$$
\left(\frac{a}{a_{0}}\right)^{-\gamma}
$$

in these models is very steep. If $\gamma \geqslant 4$ and the limits of smallest diameters are near $a_{0}$, then the mean diameters $\overline{\bar{a}} \approx a_{0}$ may be used for the practical computation.

It is impossible to get an unambiguous and exact answer to the question of the composition and size of particles of the nebula from the computed and measured color excesses because the real conditions in nebulae do not satisfy all the limiting assumptions on which the computation was performed. Photoelectric measurements also have their limitations. At greater distances from the center the nebula is too weak, while the measurements very near the center are affected by the scattered light of the illuminating star in the telescope. The photoelectric measurement described previously was performed for a distance approximately 0.1 to $0.4 r_{1}$ from the center.

The full integration over the scattering function is impossible without the assumption of some particular models. Therefore, in the preliminary research the models with which no agreement between the observed and computed color excess $E(r)$ was found were ruled out. For this purpose the method of fitting the computed course of $E(r)$ to the observed values was used. In this method, the steepness of $E(r)$ is comsidered the most important parameter. An example of the results of such a fitting is shown in tables I and II and figure 3 for dielectric particles with a radius of $3.3 \mu$. The distribution of measured values of $E(r)$ is illustrated by the crosshatched area in figure 3 . After preliminary comparison, the spherical dielectric particles with radii of $a<0.2 \mu$ and $a>0.33 \mu$ are disregarded if the refractive indices $1.33,1.55$, and 2 are used.

Although it is not possible mathematically to represent all the influences acting on a real nebula, the model of a spherical cloud where there is single scattering, at least at greater distances from the nebula, can be regarded as sufficiently satisfactory. The results obtained justify 
the conclusion that the model containing particles of a dielectric nature with a refractive index of 2 and a radius of $0,3 \mu$ is the most satisfactory for NGC 7023. The interpretation concerning particles with refractive index 1.33 and $a=0.2 \mu$ is somewhat questionable since for this model it was necessary that the nebula have larger dimensions than follows from the apparent observable radius. However, this refractive index and particle size is in good agreement for NGC 2261.

These investigations are suitable for the qualitative delimitation of probable sizes of particles. The sizes found in this investigation are somewhat larger than the supposed sizes of particles ascertained from the interstellar absorption.

TABLE I. - Results of Fitting Observed Color Excess $\mathbf{E}(\mathrm{r})$ in Reflection Nebulae on Theoretical Values NGC 7023

$$
[a=0.33 \mu]
$$

\begin{tabular}{|c|c|c|c|}
\hline \multirow{2}{*}{ Model } & \multicolumn{3}{|c|}{ Type of agreement for refractive index of - } \\
\hline & 1.33 & 1.50 & 2.0 \\
\hline $\mathbf{A}$ & $\begin{array}{l}\text { Good } \\
\text { - if } r_{1} \text { of nebula is } \\
\text { larger than } \\
\text { observed }\end{array}$ & Poor & Very good \\
\hline B & Poor & None & Good \\
\hline $\mathrm{C}^{-}$ & None & None & None \\
\hline D & Poor & None & Poor \\
\hline
\end{tabular}

TABLE II. - Comparison of Color Distribution Between Computed Models and Observations

$[\text { Diameter of nebula }=18 \text { minutes of arc }]^{\mathrm{a}}$

\begin{tabular}{|c|c|c|c|c|}
\hline \multirow{2}{*}{$a, \mu$} & \multirow{3}{*}{$\begin{array}{c}\text { Refractive } \\
\text { index } m\end{array}$} & \multicolumn{3}{|c|}{$\begin{array}{c}\text { Correlation of color distribution between computations } \\
\text { and cbservations for model - }\end{array}$} \\
\cline { 3 - 5 } & & A & C & D \\
\hline 0.30 & 2 & Good & None & Good \\
.18 & 2 & Poor & None & Poor \\
.30 & 1.55 & Poor & Good & Poor \\
.18 & 1.55 & None & None & None \\
.30 & 1.33 & None & None & None \\
.18 & 1.33 & None & Good & None \\
.30 & 1.33 & Good & None & Good \\
\hline
\end{tabular}

a In last row, adopted diameter of nebula extended to $27^{\prime}$. 


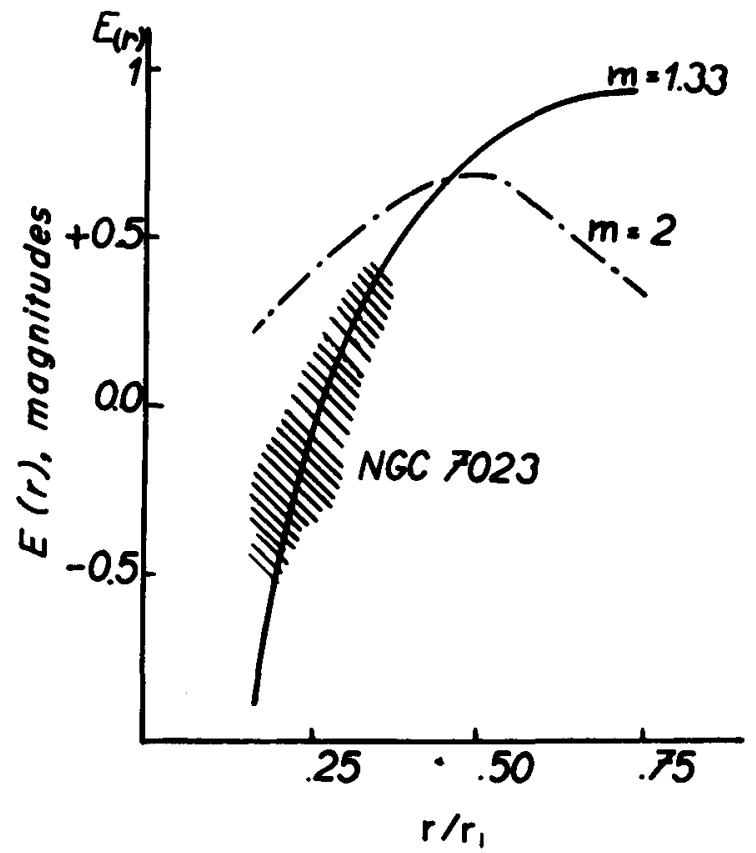

FIGURE 3. - Color excess as function of $r / r_{1}$ for refractive indices $m$ of 1.33 and 2 .

\section{REFERENCES}

1. Keenan, P. C.: Photometry of the Diffuse Nebula NGC 7023. Astrophys. J., vol. 84, 1936, p. 600.

2. Collins, O. C.: Color Indices of Reflection Nebulae. Astrophys. J., vol. 86, 1937, p. 529.

3. Henyey, L. G.; and Greenstein, J. L.: The Theory of the Colors of Reflection Nebulae, Astrophys. J., vol. 88, 1938, p. 580.

4. Johnson, H. M.: Photoelectric Photometry of Diffuse Galactic Nebulae and Comet Arend-Roland. Astron. Soc. Pacific, Pub., vol. 72, 1960, p. 10.

5. Vanýsex, V.; and Svatoš, J.: Solid Particles in Reflection Nebulae I. Acta Univ. Carol. No. 1, 1964.

6. Katchikian, E.: Akademiia Nauk Armianskoi SSR, Izvestiia, Seriia Tekhnicheskikh Nauk. vol. 10, No. 5, 1957.

7. Martel, T. M.: Polarisation et Coleur des Nébuleuses Diffusantes. Ann. Astrophys., Suppl. 7, 1958.

8. Hall, R. C.: Polarization and Color Measures of NGC 2261. Astron. Soc. Pacific, Pub., vol. 77, 1965, p. 158.

\section{DISCUSSION}

Wickramasinghe: Why is there such a sharp discontinuity of trend between $m=1.33$ and $m=1.55$ in table II?

Vanýsek: The refractive index is very important because an increase in its value produces a shift of nebular diameter. I think these results 
are very similar to those of the previous paper, but the geometric configuration is different. I believe that our geometry is much closer to that of the real nebula.

Lind: Could the diameter of the cloud affect the results?

Vanysek: We say that the diameter of the whole nebula is 1 and we take into consideration the fact that the nebula has a diameter (effective diameter) of about 18 minutes of arc.

Lind: You didn't change the diameter then?

Vanysek: If we take into consideration that the nebula itself is much greater than the observed diameter, 25 or 75 percent greater, we can find good agreement between the theory and the observation if the index of refraction is 1.33 and the diameter of the particles is about $0.3 \mu$.

Greenberg: I think that what is bothering all of us is a lack of continuity in the sense that one would expect to get progressively better or progressively worse results within a particular framework if the value of $\boldsymbol{m}$ is increased from 1.33 to 1.55 to 2 . What bothers us is the fact that the trend is not monotonic. However, it may well be that this is just some property of this type of geometry.

Vanysek: I think that our main problem is that we made all the computations for spherical particles with real indices only. I am not sure what the effect would be of introducing an imaginary part to the refractive index smaller or larger than 1 or of using other types of particles. The experimental measurements of real clouds of small particles as made many years ago by Richter are not representative enough because they were for very large particles. I think that the experimental measurements Dr. Greenberg has in mind for ellipsoids may be useful for reflection nebulae computations. 